\title{
Ventricular Tachycardia/Fibrillation Detection Algorithm for 24/7 Personal Wireless Heart Monitoring
}

\author{
Steven Fokkenrood*, Peter Leijdekkers**, Valerie Gay** \\ University of Technology, Faculty of IT, \\ PO Box 123 Broadway NSW 2007, Australia \\ * s.a.w.fokkenrood@student.utwente.nl, ${ }^{* *}$ \{peterl, valerie\}@it.uts.edu.au
}

\begin{abstract}
This paper describes a Ventricular Tachycardia/Fibrillation (VT/VF) detection algorithm that is specifically designed for a 24/7 personal wireless heart monitoring system. This monitoring system uses Bluetooth enabled biosensors and smart phones to monitor continuously cardiac patients' vital signs. Our VT/VF algorithm is optimized for continuous real-time monitoring on smart phones with a high sensitivity and specificity. We studied and compared existing VT/VF algorithms and selected the one which suited best our requirements. However, we modified and improved the existing algorithm for the smart phone to achieve better performance results. We tested the algorithm on full-length signals from the physionet CU, MIT-db and MIT-vfdb databases [16] without any pre-selection of VT/VF or normal QRS-complex signals. We achieved $97 \%$ sensitivity, $98 \%$ accuracy and $98 \%$ specificity for our implementation which is excellent compared to existing algorithms.
\end{abstract}

Keywords: ventricular/tachycardia algorithm, ECG signal processing, heart monitoring, mobile-health, wireless ECG sensors.

\section{Introduction}

Cardiovascular diseases are now the number one cause of death in the United States and most European countries. The costs of medical help for cardiovascular patients is already estimated to be $€ 316$ billion in the United States in 2006 [1]. Using state-of-the-art sensor technology and smart phones, it is now possible to lower the costs related to heart patients. The latest ECG monitors are now wearable and reliable, allowing the patient to wear them 24/7. The use of mobile health applications to monitor patients is a booming business worldwide. The market is estimated to be $\$ 81$ billion (USD) in the United States alone.

At the University of Technology, Sydney, we work on a context-aware personal health monitoring project [2]. In this project we use smart phones and wearable Bluetooth bio-sensors for patient monitoring and rehabilitation. When a life threatening situation is detected, the smart phone automatically calls and SMSes preprogrammed phone numbers indicating the location and reason for the emergency 
call. Also potential bystanders are notified by a message played continuously on the smart phone and instruct them what to do.

This paper focuses on one fundamental part of this project: the detection of Ventricular Fibrillation (VF) which is the main cause of sudden cardiac death and Ventricular Tachycardia (VT). Ventricular Tachycardia is a very rapid beating of the heart, resulting in a diminished cardiac output. Our objective is to implement an efficient VT/VF detection algorithm for a mobile device which should satisfy the following requirements:

- It must be able to process the ECG data in real-time on the smart phone. To save battery power we need to avoid unnecessary processing on the smart phone.

- The algorithm should detect VT/VF with high sensitivity and specificity. We want to minimize the possibility of missing a VT/VF episode which can be lethal.

- Minimise false alarms. The classification of a QRS complex as VT/VF (i.e. false classification) is inconvenient for the patient using the monitoring system.

- We do not need to distinguish between VT and VF since it is not meant to be used in defibrillators to decide whether an arrhythmia is shockable.

Numerous algorithms exist to detect VT and VF and we evaluated them against our requirements. We also investigated the triggers for a VT/VF onset in order to improve the algorithm. Public online databases are used to test our algorithm and we compared the performance of our algorithm against other algorithms [3 - 9] using the same dataset.

This paper is organised as follows. Section 2 evaluates existing VT/VF algorithms and identifies those that are a good basis for our project. Section 3 discusses the design and implementation of our improved VT/VF algorithm. Section 4 presents the performance results and section 5 shows how the VT/VF algorithm is integrated in the smart phone application. Finally, section 6 concludes this paper.

\section{Evaluation of publicly available VT/VF algorithms}

Two factors are of particular importance which are high sensitivity and specificity. The sensitivity is a measure for how accurate the algorithm detects VT/VF episodes in an ECG. Specificity corresponds to the algorithm's capability to alarm if, and only if, it is an actual VT/VF signal.

In addition limited processing power of the smart phone implies restrictions on the possible algorithms for implementation since the smart phone has limited processing capabilities (typically a 200-500 $\mathrm{MHz}$ processor). Algorithms that are not practical to implement on a mobile device are Fuzzy Neural Network (FNN) designs or Wavelet Transformation (WTF) based algorithms. A FNN algorithm is often implemented on high end processors which are not yet available for smart phones [4]. WTF algorithms are very CPU intensive which is not practical for mobile phones and will drain the battery quickly [4]. 
The smart phone provides an ambulatory monitoring environment for the patient. The patient is able to move freely while being continuously monitored. We selected a modified lead II type ECG sensor with only two electrodes attached directly to the sensor. Using a 2-lead sensor implies that we look at algorithms that use the physionet databases MLII signals and omitted those that use their own intracardiac ECG dataset [3].

Several algorithms claim high sensitivity and specificity, but on closer inspection many algorithms use pre-selections of ECG database signals to evaluate their performance [5] or pre-select MLII input signals [6-8]. Algorithms tested on partial signals are; Threshold Crossing Interval (TCI) [7], Complexity Measure (CM) [8], Modified Complexity Measure (MCM) [5] and the Auto-Correlation Function $\left(\mathrm{ACF}_{95 / 99}\right)$ [6]. $\mathrm{ACF}_{95}$ and $\mathrm{ACF}_{99}$ refer to Fisher statistics degree of freedom.

Table 1 shows the results of the algorithms on pre-selected signals as published in [5-8], as well as, the results of the same algorithms tested on full-length signals [4]. In case of full-length signals the performance of the algorithms drops significantly. The TCI algorithm is generally regarded as a good algorithm [4] for VT/VF detection and reaches $75.1 \%$ sensitivity. However, it classifies any heart rate higher than $150 \mathrm{bpm}$ as VT or VF which is an incorrect assumption since a healthy person can easily have a heart rate of $150 \mathrm{bpm}$ and higher when exercising.

Table 1. Performance results (7stw $=7$-second time window)

\begin{tabular}{|lllll|}
\hline Algo & \multicolumn{2}{c}{ Pre-selected signals } & \multicolumn{2}{c|}{ Full length signals (8stw, [4]) } \\
\hline & Sensitivity (\%) & Specificity (\%) & Sensitivity (\%) & Specificity (\%) \\
\hline TCI & $100(7 \mathrm{stw},[7])$ & N/A & 75.1 & 84.4 \\
\hline CM & $100(7 \mathrm{stw},[8])$ & $100(7 \mathrm{stw},[8])$ & 59.2 & 92.0 \\
\hline MCM & 89.8 [5]) & N/A & 51.2 & 84.1 \\
\hline ACF $_{95}$ & N/A & N/A & 49.6 & 49.0 \\
\hline ACF $_{99}$ & $100(4.5 s t w, ~[6])$ & $100(4.5 s t w, ~[6])$ & 69.2 & 35.0 \\
\hline SPEC & $93(8 \mathrm{stw},[9])$ & $79(8 \mathrm{stw},[9])$ & 29.1 & 99.9 \\
\hline VF & 91 & 94 & 18.8 & 100 \\
\hline ADA & & $95.9(10 \mathrm{stw},[9])$ & $94.4(10 \mathrm{stw},[9])$ \\
\hline SCA & & $71.2(8 \mathrm{stw},[4])$ & $98.5(8 \mathrm{stw},[4])$ \\
\hline QRC & & $91.9(6 \mathrm{stw},[12])$ & $98.3(6 \mathrm{stw},[12])$ \\
\hline
\end{tabular}

Jekova describes in [9] the performance results for the Spectral analysis (SPEC) [10] and VF-Filter (VF) [11] algorithms on pre-selected MLII signals. These algorithms perform very well with respect to specificity but sensitivity is very low for full-length signals for both algorithms.

The Amplitude Distribution Analysis (ADA) algorithm [9], Signal Comparison Algorithm (SCA) [4] and QRS detection and Rhythm Classification algorithm (QRC) [12] use full-length signals to test their performance. ADA applies noise detection, asystole detection, VT/VF detection and beat detection to classify a heart signal. SCA compares a heart signal interval with four different reference signals to classify a heart signal episode (i.e. three QRS-complexes and one VT/VF reference signal). The 
residual between the measured signal and the four reference signals is a measure for classification. ADA, SCA and QRC are tested on full-length signals from the MIT-db, MIT-vfdb, AHA and CU databases. The advantage of the ADA, QRS and SCA algorithms is the use of several steps to classify a heart signal. This improves the performance considerably compared to other algorithms.

From our literature study we found out that the SCA algorithm is not a practical solution for our application since it will be very hard to obtain a VT and VF reference signal for each patient. We agree with Fernandez about the QRC algorithm where he states that "System failed, in general, when signal, even filtered, holds noisy and the noise peaks had very big amplitude" [5, 12]. It will fail in an ambulatory environment for our 2-lead sensor. The ADA algorithm is the best candidate for implementation on the smart phone where beat detection and classification is performed together with VT/VF detection [5, 12].

\section{Improved VT/VF Algorithm}

We modified the original ADA algorithm and altered the order of the signal processing routines. We placed the beat detector before the VT/VF classification as suggested by Fernandez in [12]. This improves the overall performance of the algorithm since the beat detector is less CPU intensive compared to the VT/VF routine. Fig. 1 shows the improved ADA algorithm as implemented for the mobile device.

Beat detector:

ECG data is collected from the 2-lead ECG sensor or physionet ECG files. We use the open source beat detector from Hamilton [13] to obtain the heart rate and beat type which can be QRS, Premature Ventricular Contraction (PVC) or Unknown. Based on the heart rate and/or beat type we check for VT/VF for the following conditions:

- Heart rate lower or higher than the threshold set by a cardiologist. The thresholds are patient specific and might indicate something abnormal for that patient [14].

- High Heart Rate Variability (HRV) can indicate the onset of VT and VF [14].

- High number of PVC’s within a certain time limit can indicate a VT onset [14].

- Many 'unknown' beat types in a short time interval indicate that something is wrong with the heart signal because QRS-complexes cannot be detected. 
Fig. 1. Flow chart for the modified ADA algorithm

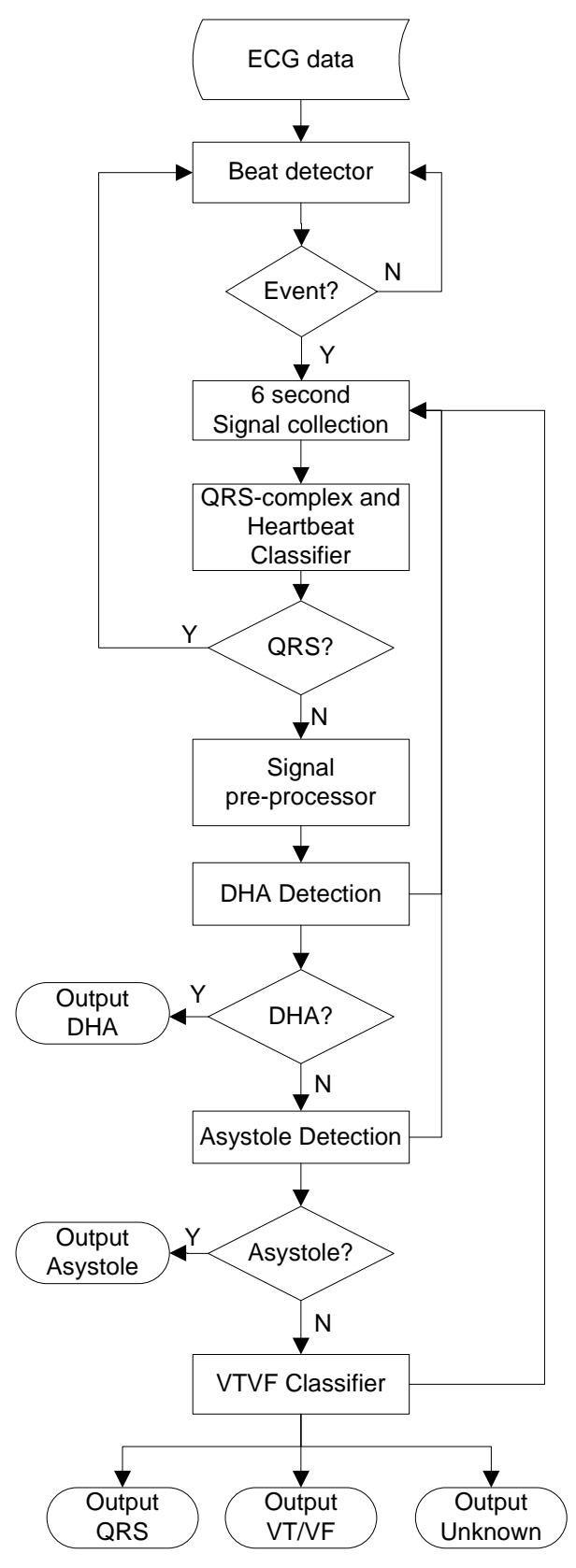


6 second signal collection

The algorithm will collect 6 seconds of ECG data for further analysis if one of the above mentioned events occurs. From simulation studies conducted with Matlab we determined that 6 seconds is sufficient to analyse a signal efficiently. Signal classification is based on statistical distributions which needs a sufficient amount of signal samples. A longer time window forces the algorithm to classify a signal collection possibly containing both QRS and VT/VF signals as either QRS or VT/VF which is incorrect. On the other hand, a too short time window will result in the algorithm not being able to classify a signal correctly and will return an 'unknown' result. We also collect the heart rate, beat types and average heart rate for this 6 second interval.

\section{QRS-complex and heartbeat classifier}

This routine checks for high HRV and the number of PVCs or 'unknown' heart beats in the 6 second signal. Further processing of the signal is only required, if:

- The HRV remains high during this 6 second interval.

- The number of detected PVC's or 'unknown' beat types is higher than $50 \%$ of the detected beat types.

Signal pre-processor

In case of an expected arrhythmia the 6 second signal is filtered as follows:

- The signal is normalized, removing the gain of the signal. Gain removal allows millivolt $(\mathrm{mV})$ thresholds for the DHA detector and Asystole classifier.

- The signal is then forward-backward filtered though a $1.4 \mathrm{~Hz}$ high-pass filter. This removes the low frequency noise causing offset and drift of the ECG signal.

- The signal is then forward-backward filtered through a low-pass filter. This removes the high frequency noise from muscle movement using a $30 \mathrm{~Hz}$ low-pass filter [3, 4, 12]. Forward-backward filtering of the signal ensures that there is no amplitude distortion and phase shifting of the signal.

\section{DHA detection}

We then check the signal for Dangerous Heart Activity (DHA). DHA can be a noisy ECG signal or an arrhythmic VT or VF signal. Large amplitude differences may imply a noisy signal but if there are too many oscillations it can be a VF signal since fast contractions of the ventricles result in high oscillating skew rates in the ECG signal. A higher amplitude of the current epoch compared to the previous 6 second epoch is used to ignore unreadable ECG signal periods. When DHA is detected the algorithm will automatically analyse the following 6 second epoch.

Asystole Detection

In the absence of large oscillations, the epoch is then analysed for a low amplitude signal. Asystolic signal cannot be analysed with the normal VT/VF classifier because of the low amplitude. If the amplitude is below $0.3 \mathrm{mV}$ we will classify the signal with the asystole classifier. If not, we use the VT/VF classifier to determine the type of heart signal. 
Asystole Classifier

When an asystole is suspected we use an asystole classifier to determine the signal type (i.e. QRS, Asystole or VT/VF). We determine the following characteristics of the signal:

- We calculate the number of peaks in the signal. This is a rough indicator for the heart rate.

- The relation between the samples above and below the mean signal. For QRS signal this relation is lower than for VT/VF signal [12].

- The overall amplitude of the signal.

The signal is regarded asystolic when the maximum amplitude does not cross the $0.1 \mathrm{mV}$ threshold. The signal will be classified as a QRS complex if less than $40 \%$ of the signal is above half the maximum value, and the number of possible QRS complexes is below the emergency heart rate. Otherwise, it will be classified as a VT/VF signal. The Asystole classifier will trigger the algorithm to automatically analyse the following 6 second epoch.

\section{VT/VF Classifier}

When the signal epoch is not classified as asystole by the Asystole detector we then process it by the VT/VF classifier. This algorithm is based on statistical distribution of QRS and VT/VF signal samples as proposed by Jekova [9]. For each 6 second interval (I), three amplitude ranges are defined, and signal samples $\left(\mathrm{S}_{\mathrm{i}}\right)$ falling within a certain amplitude range for this interval are added, which are shown in equation 1.

$$
\begin{gathered}
T_{1}=\left(0.8 \cdot \max (|I|) \leq S_{i} \leq \max (|I|)\right) \\
T_{2}=\left(|\bar{I}| \leq S_{i} \leq 0.95 \cdot \max (|I|)\right) \\
T_{x}=\left((|\bar{I}|-M D) \leq S_{i} \leq(|\bar{I}|-M D)\right), \text { with } M D=\frac{1}{n} \sum\left|S_{i}-\bar{I}\right| \\
T_{3}=\frac{T_{1} \cdot T_{2}}{T_{x}}
\end{gathered}
$$

The result of the VT/VF classifier depends on the signal samples accrued for $T_{1}$, $\mathrm{T}_{2}$ and $\mathrm{T}_{3}$, which are based on Jekova's algorithm [9]. Classification of the signal is presented in equation 2. An epoch processed by the VT/VF classifier will always trigger the algorithm to analyse the next 6 second epoch even if the result is a QRS complex. 


$$
\begin{gathered}
\text { QRS }=\left(T_{1}<120 \& T_{2}<456 \& T_{3}<100\right) \mathrm{V} \\
\left(120<T_{1}<192 \& T_{2}<288 \& T_{3}<100\right) \\
V T / V F=\left(T_{1}<120 \& T_{2} \geq 456\right) \mathrm{V} \\
\left(T_{1} \geq 120 \& T_{3} \geq 100\right) \mathrm{V}\left(T_{2} \geq 528\right) \\
\text { Unknown }=\text { Everything else }
\end{gathered}
$$

\section{Performance results}

We implemented the algorithm on a Microsoft Windows Mobile Pocket PC platform using the $\mathrm{C \#}$ programming language. The algorithm is tested on the following full-length signals from the online physionet databases [15, 16]:

- CU files: CU01-CU35.

- $\quad$ MIT-db files: 100-119, 121-124, 200-203, 205, 207-215, 217, 219-223, 228, 230234.

- $\quad$ MIT-vfdb files: 418-430, 602, 605, 607, 609-612, 614, 615.

We did not modify or pre-select any of the signals. The MIT-db and MIT-vfdb files contain MLII and V5 signals but we only used the MLII signal since our ECG sensor is a 2-lead MLII type sensor. The results of the algorithm are represented by four parameters as shown in equation 3.

$$
\begin{gathered}
S e=\frac{T P}{T P+F N} \quad S p=\frac{T N}{T N+F P} \quad P P=\frac{T P}{T P+F P} \\
A c c=\frac{T P+T N}{T P+F P+T N+F N}
\end{gathered}
$$

- $\quad$ True positive (TP) is the correct classification of a VT or VF signal.

- True negative (TN) is the correct classification of a normal heart signal.

- False positive (FP) is the incorrect classification VT/VF while the heart signal is normal.

- False negative (FN) is the incorrect classification of a QRS complex while the heart is in a VT/VF state.

Sensitivity (Se) indicates the capability to detect all occurring VT/VF episodes whereas Specificity (Sp) signifies the discriminating quality of the algorithm.

Positive Predictivity (PP) indicates the probability that a signal epoch classified as $\mathrm{VT} / \mathrm{VF}$ is truly VT/VF. It is a measure for the inconvenience to the patient, since it 
represents the ratio between actual VT/VF arrhythmia and false alarms. Accuracy (Acc) specifies all the correct decisions made by the algorithm.

The output of the algorithm can be QRS, DHA, Asystole, VT/VF or Unknown and the time of occurrence. We manually compared the output of the algorithm with the physionet ECG annotations. Automatic comparison is not possible since the format of the annotation files vary per database. In order to relate the annotations with the algorithm output we interpreted the algorithm output as follows:

- DHA output is classified as TP or FN. The reason being that DHA could be a dangerous rhythm.

- Unknown output is always an incorrect classification which results in FP for a QRS signal, and FN for a VT/VF signal.

- If the physionet annotations define a signal interval as noise, unreadable or asystole, the output of the algorithm cannot be classified as TP, FP, TN or FN. These heart signals do not contain useful information and therefore not contribute to the performance result of the algorithm.

- Asystole output does also mean that the heart signal does not contain useful information. The amplitude of the signal is too low to detect any QRS or arrhythmia characteristic. Therefore, this output is not classified as TP, FP, TN or FN.

- The algorithm can only analyse and classify 6 second intervals. If the physionet annotation corresponds with the output within this 6-second interval it is used as a valid result.

The results are presented in Table 2 .

Table 2. Performance results

\begin{tabular}{|lllll|}
\hline & CU & MIT-vfdb & MIT-db & Overall \\
\hline Sensitivity & $95 \%$ & $91 \%$ & $100 \%$ & $97 \%$ \\
\hline Specificity & $96 \%$ & $96 \%$ & $99 \%$ & $98 \%$ \\
\hline Positive predictivity & $81 \%$ & $85 \%$ & $65 \%$ & $73 \%$ \\
\hline Susceptibility & $96 \%$ & $96 \%$ & $99 \%$ & $98 \%$ \\
\hline Accuracy & $95 \%$ & $96 \%$ & $99 \%$ & $98 \%$ \\
\hline
\end{tabular}

The overall sensitivity performance of $97 \%$ corresponds to the recognition of every single VT/VF epoch. But we actually detect $100 \%$ of the VT/VF episodes as long as they are longer than 6 seconds. If VT/VF episodes are shorter than 6 seconds we do not classify them as VT/VF since the heart returns to a normal state and in our situation we do not need to raise an alarm.

The overall specificity of $98 \%$ indicates that the algorithm will raise an alarm in case of a real VT/VF signal in $98 \%$ of the cases. The remaining few cases $(2 \%)$ correspond to unreadable parts of a signal. If the signal is unreadable we should alarm the user so that he/she can check the ECG sensor. 
Positive predictivity is not a good indication for the number of false alarms. A better indicator would be the amount of false positive classifications compared to the official number of QRS epochs. This indicator is called Susceptibility and corresponds to the amount of FP classifications during a QRS signal, see equation 4. It is a more accurate measure for the number of false alarms. If we apply this equation for our algorithm, we achieve $98 \%$ susceptibility.

$$
S u=1-\frac{F P}{T N}
$$

\section{Integration with the Personal Health Monitor Application}

We integrated the algorithm with the personal health monitor application and tested its functionality using the MIT-db, MIT-vfdb and CU databases. The application has a demo-mode functionality where pre-recorded (physiobank) ECG files can be processed by the health monitor application. The application processes the physiobank signals and live ECG data in the same manner. The only difference is that in the demo-mode the actual alarm functionality (i.e. sending SMS and call) is disabled. In non demo-mode the application generates an alarm to notify the user when the algorithm detects a dangerous arrhythmia (i.e. VT/VF, Asystole or DHA). If the user does not react in a certain time the application will automatically call and SMS pre programmed phone numbers stating the reason of the call and the current location of the patient.

Fig. 2. Detecting VT/VF and raise an alarm
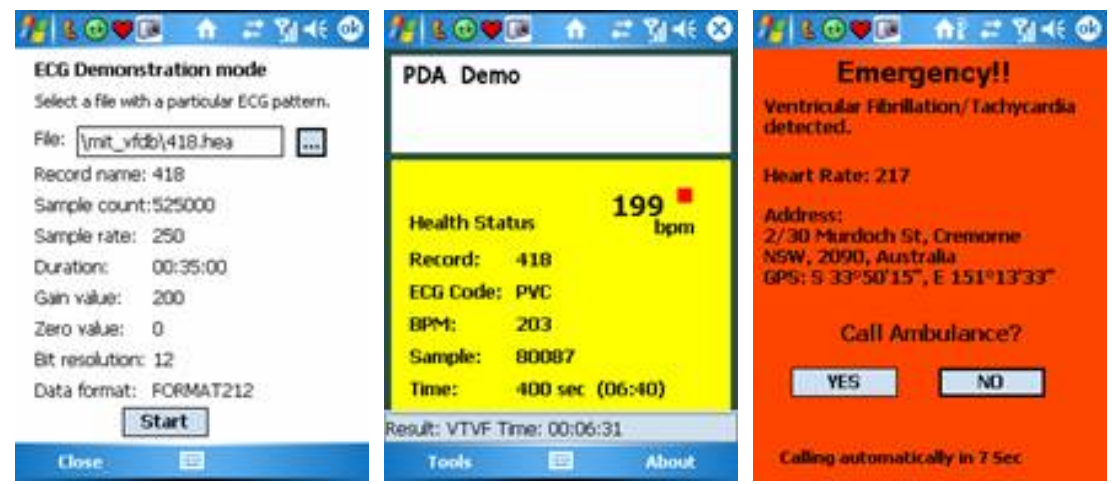

We tested the performance of the algorithm on two different smart phones (I-mate ${ }^{\circledR}$ JASJAR, 520MHz processor and i-mate ${ }^{\circledR}$ K-JAM, $195 \mathrm{MHz}$ processor). Both smart phones are able to process the physionet ECG data files in real-time. Tests with the Alive ECG sensor showed no problem processing ECG data in real-time. 


\section{Conclusion}

The heart monitoring algorithm implemented for the smart phone is able to detect VT/VF, Asystole and dangerous heart activity (DHA) with high sensitivity and specificity. We tested the algorithm on full-length signals without any pre-selection of VT/VF or normal QRS-complex signals. We achieved 98\% accuracy, 97\% sensitivity and $98 \%$ specificity which is an excellent score compared to existing algorithms and suitable for the personal health monitor application on the PDA.

We have also put mechanisms in place to deal with the few false alarms allowing the patient to disable the alarm. An ECG signal is recorded automatically as soon as it is identified as abnormal which allows further examination by the cardiologist. The patient can also start recording if he/she feels an abnormal heart rate or rhythm This means that our 24/7 Personal Wireless Heart Monitoring System can adequately be used to detect VT/VF episodes.

Time is a crucial factor when VT/VF is detected. For the patient to have a chance to survive VT/VF, a defibrillator should be applied within 5 minutes. Our algorithm detects the onset of VT/VF within 6 seconds. It will raise an alarm if another VT/VF epoch is detected to avoid false alarms. This means that after 12 seconds of a VT/VF onset, emergency services and caregivers are notified via SMS and automatically placed phone calls. This will increase the chance that help can be given in time.

Point of interest is when we want to raise an alarm. An alarm can be raised after the first VT/VF detection or additional VT/VF classifications can be required. This depends on the situation of the patient. For example, if the patient is in a nursing home we could alarm the medical staff as soon as we have a VT/VF epoch detection. If the patient lives alone, we would call the emergency services after two 6 second VT/VF epochs to reduce false alarms. Whatever the answer is, our application can be personalised to suit the situation.

The next step is to improve the algorithm to detect earlier signs of heart failure and other arrhythmia associated with cardiovascular diseases such as Atrial Fibrillation. This will allow us to identify signs of an upcoming cardiac arrest or identify abnormalities and therefore able to warn the patient or caregiver earlier.

\section{References}

1. AHA. Cardiovascular Disease Cost. Last accessed 3 April 2007 [cited; Available from: http://www.americanheart.org/].

2. Leijdekkers, P., Gay, V., Personal Heart Monitoring and Rehabilitation System using Smart Phones. 2006.

3. Throne, R.D., Janice M. Jenkins, Lorenzo A. Dicarlo, A Comparison of Four New Time Domain Techniques for Discriminating Monomorphic Ventricular 
Tachycardia from Sinus Rhythm using Ventricular Waveform Morphology. IEEE Transactions on Biomedical Engineering, 1991. Vol. 38: p. 561 - 570.

4. Amann, A.R.T., Unterkofler, K., Reliability of Old and New Ventricular Fibrillation Detection Algorithms for Automated External Defibrillators. BioMedical Engineering Online, 2005. 4: p. 1 - 23.

5. Ayesta, U.L.S., Romero, I., Complexity Measure Revisited: A New Algorithm for Classifying Cardiac Arrhythmias. IEEE Explorer, 2001. 2: p. 1589 1591.

6. Chen, S., Thakor, N.V., Mower, M.M., Ventricular Fibrillation Detection by a Regression Test on the Autocorrelation Function. Medical and Biological Engineering and Computing, 1987. Vol. 25: p. 241 - 249.

7. Thakor, N., Yi-Zheng Shu, V., Kong-Yan Pan, Ventricular Tachycardia and Fibrillation Detection by a Sequential Hypothesis Testing. IEEE Transactions on Biomedical Engineering, 1990. Vol. 37: p. 837 - 843.

8. Zhang, X.-S. Yi-Sheng Zhu, Nitish V. Thakor and Zhi-Zhong Wang, Detecting Ventricular Tachycardia and Fibrillation by Complexity Measure. IEEE Transactions on Biomedical Engineering, 1999. Vol. 46: p. 548 - 555.

9. Jekova, I., Krasteva, V., Real Time Detection of Ventricular Fibrillation and Tachycardia. Physiological Measurements, 2004. Vol. 25: p. 1167 - 1178.

10. Barro, S., Ruiz, R., Cabello, D., and Mira, J., Algorithmic Sequential Decision-making in the Frequency Domain for Life Threatening Ventricular Arrhythmias and Imitative Artefacts: a Diagnostic System. Journal of Biomedical Engineering, 1989. 11: p. 320 - 328.

11. Kuo, S.D.R., Computer Detection of Ventricular Fibrillation. Computers in Cardiology, 1978. IEEE Computer Society: p. 347 - 349.

12. Fernandez, A.R., Folgueras, J., Colorado, O., Validation of a Set of Algorithms for Ventricular Fibrillation Detection: Experimental Results. in Proceedings of the 2th Annual International Conference of the IEEE EMBS. 2003. Mexico.

13. Hamilton, P.S., Tompkins, W. J. , Evaluation of QRS Detection Algorithms Using the IBM PC. Engineering Medical Biological Society, 1985. Annual Conference of the IEEE: p. 830 - 833.

14. Nemec, J., Hammill, S.C. ,Shen, W.K., Increase in Heart Rate Precedes Episodes of Ventricular Tachycardia and Ventricular Fibrillation in Patients with Implantable Cardioverter Defibrillators: Analysis of Spontaneous Ventricular Tachycardia Database. Scientific Congress of NASPE, 1999. Vol. 22: p. 1729 - 1738.

15. Goldberger, A.L., Amaral, L.A.N. ,Glass, L., Hausdorff, J.M, Ivanov P.Ch, Mark, R.G., Mietus, J.E., Moody, G.B., Peng, C.-K, and Stanley, H.E., PhysioBank, PhysioToolkit, and PhysioNet Components of a New Research Resource for Complex Physiologic Signals. Circulation, 2000. 101: p. e215 e220.

16. MIT-BIH. CU Ventricular Tachyarrhythmia Database. 1992 [cited; Available from: http://www.physionet.org/]. 